\title{
Prognostic Factors and Management of Colorectal Gastrointestinal Stromal Tumors
}

\author{
Nuno Rafael Pereira Reis ${ }^{1}$ (1) Laura Elisabete Ribeiro Barbosa $^{1,2}$ (이 \\ ${ }^{1}$ Department of Surgery and Physiology, Faculdade de Medicina, \\ Universidade do Porto, Porto, Portugal. \\ ${ }^{2}$ Department of General Surgery, Centro Hospitalar Universitário São \\ João, Serviço de Cirurgia Geral, Porto, Portugal. \\ Address for correspondence Nuno Rafael Pereira Reis, PhD, Alameda \\ Professor Hernâni Monteiro, 4200-319, Porto, Portugal \\ (e-mail: nunorafa.reis@gmail.com). \\ J Coloproctol 2022;42(1):68-76.
}

\begin{abstract}
Introduction The gastrointestinal stromal tumor (GIST) is the most common mesenchymal neoplasm of the gastrointestinal tract. Even though it can be found in any location of the digestive tract, the colorectal GIST is rare. With this study, we aim to review the current knowledge regarding the prognosis and management of colorectal GIST.

Methods A literature search was conducted in PubMed, and 717 articles were collected. After analyzing these studies, 60 articles were selected to use in this review. Results The mitotic index, as well as tumor size and location were identified as good discriminators of prognosis in various studies. Surgery remains the only curative therapy for potentially resectable tumors. However, even after surgical resection, some patients develop disease recurrence and metastasis, especially those with highrisk tumors. Therefore, surgical resection alone might be inadequate for the management of all colorectal GISTs. The discovery of GIST's molecular pathway led to a shift in its therapy, insofar as tyrosine kinase inhibitors became part of the treatment schemes for this tumor, revolutionizing the treatment's outcome and prognosis.

\section{Keywords}

- gastrointestinal stromal tumors

- colorectal neoplasms

- therapeutics

- prognosis

Discussion/Conclusion The controversy concerning colorectal GIST prognosis and treatment can be, in part, attributed to the limited number of studies in the literature. In this review, we gathered the most recent knowledge about the prognosis and management of GIST in this rare location and propose two algorithms for its approach. Lastly, we highlight the importance of an individualized approach in the setting of a multidisciplinary team.
\end{abstract}

\section{Introduction}

The gastrointestinal stromal tumors (GISTs) are the most common mesenchymal tumors of the gastrointestinal (GI) tract. Despite this, they are rare tumors, comprising only $0.2 \%$ of all gastrointestinal malignancies. ${ }^{1,2}$

received

March 25, 2021

accepted after revision

August 6, 2021

published online

January 31,2022
DOI https://doi.org/ 10.1055/s-0041-1740297. ISSN 2237-9363.
Gastrointestinal stromal tumors originate in the submucosal layer of the GI tract, ${ }^{3}$ and most of them are located in the stomach (60\%) or small intestine (30\%), even though they can be found in any part of the digestive tract. ${ }^{1}$ Only $5 \%$ of GISTs are located in the rectum, and $1 \%$ are colonic, found primarily in the transverse and sigmoid colon, making the

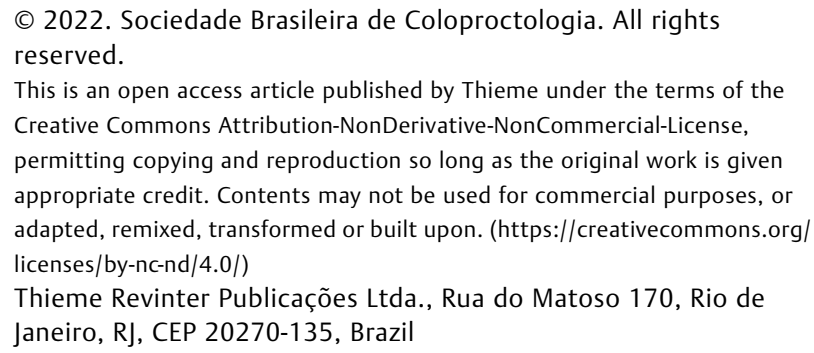

This is an open access article published by Thieme under the terms of the Creative Commons Attribution-NonDerivative-NonCommercial-License, permitting copying and reproduction so long as the original work is given appropriate credit. Contents may not be used for commercial purposes, or adapted, remixed, transformed or built upon. (https://creativecommons.org/ licenses/by-nc-nd/4.0/) Thieme Revinter Publicações Ltda., Rua do Matoso 170, Rio de Janeiro, RJ, CEP 20270-135, Brazil 
colorectal GIST a rare entity. ${ }^{3-5}$ In fact, they account for only $0.1 \%$ of all colorectal malignancies. ${ }^{6}$ Additionally, GISTs located in the esophagus, greater omentum and mesentery are even rarer. ${ }^{1}$

Studies show that GISTs arise from the interstitial cells of Cajal, which can be found in the myenteric and submucosal plexus of the GI tract. ${ }^{7,8}$ Most of these neoplasms are driven by the gain-of-function mutations in the c-Kit (75\%) or the platelet-derived growth factor receptor $\alpha$ (PDGFR $\alpha)$ oncogenes (10-15\%), leading to receptor tyrosine kinase (KIT or CD117) and PDGFR $\alpha$ receptor overexpression, respectively. ${ }^{7,9}$ Consequently, there is an increase in cell proliferation and survival, which leads to tumor growth and progression. ${ }^{10}$ Usually, GISTs that do not have any of the mutations mentioned above harbor mutations in less frequently involved genes such as the BRAF, NRAS, NF1, and SDH genes. $^{11,12}$

This neoplasm's clinical presentation is correlated with its location, as well as its size and speed of growth. Hence, the clinical spectrum of GISTs is very wide: they can vary from asymptomatic benign lesions detected incidentally by imageology, to extremely symptomatic fatal tumors. ${ }^{13,14}$ The signs and symptoms are usually nonspecific and include abdominal pain, bloating, GI bleeding, palpable mass, and perforation. 3,14

Approximately $20 \%$ of the patients with GIST present with metastases at the time of diagnosis. ${ }^{15}$ The tumor usually metastasizes to the liver and peritoneum. ${ }^{1}$ Metastases to lymph nodes are rare, except in pediatric and syndromic GISTs. ${ }^{1,15}$ The lung and bone are also rare sites for the occurrence of metastases ${ }^{3}$.

Usually, the GISTs are not diagnosed before pathological examination of biopsy specimens, as they are rare tumors. ${ }^{16}$ Histologically, the tumor can present with different patterns: spindle (70\%), epithelioid (20\%) or mixed (10\%) morphology. ${ }^{1,5}$ Immunochemistry is also important for GIST diagnosis and for the differential diagnosis with other neoplasms, insofar most GISTs stain for CD117, CD34, and DOG1. . $^{10,15,17-19}$ Contrast-enhanced computed tomography (CT) and magnetic resonance imaging (MRI) are also useful tools that can be used when approaching a patient with suspected GIST. ${ }^{11,17}$

Due to their rarity, few studies have focused on the management of colorectal GIST and the factors determining tumor malignancy and response to therapy. Additionally, controversy in the literature still exists. Therefore, the aim of this study is to review the current knowledge regarding the prognostic factors and management of colorectal GISTs. Moreover, we propose two algorithms for the approach of patients with localized (-Fig. 1) and unresectable/metastatic GIST (-Fig. 2).

\section{Methods}

Using the search engine PubMed, a literature search was conducted in October 2020, as follows: (Gastrointestinal Stromal Tumors [Mesh] OR "GIST") AND (colon OR rectum OR colorectal OR Colorectal Neoplasms [Mesh]) AND (treatment OR surg* OR manag* OR operati* OR Therapeutics [Mesh] OR Prognosis [Mesh] OR prognos* ${ }^{*}$. Only articles written in English or Portuguese were included, and no restriction of publication date was applied.

From this search were obtained 717 articles. The title and abstract of these studies were read and 99 articles were selected for full-text reading. The exclusion criteria were based on the availability of full text and type of article-casereports, letters, or commentaries were not selected for further reading.

Out of these 99 articles, only 50 were considered appropriate to be used in this review.

An additional search was conducted to include missing information, such as evidence regarding the diagnosis of GIST and novel prognostic factors. Therefore, 10 more articles were selected.

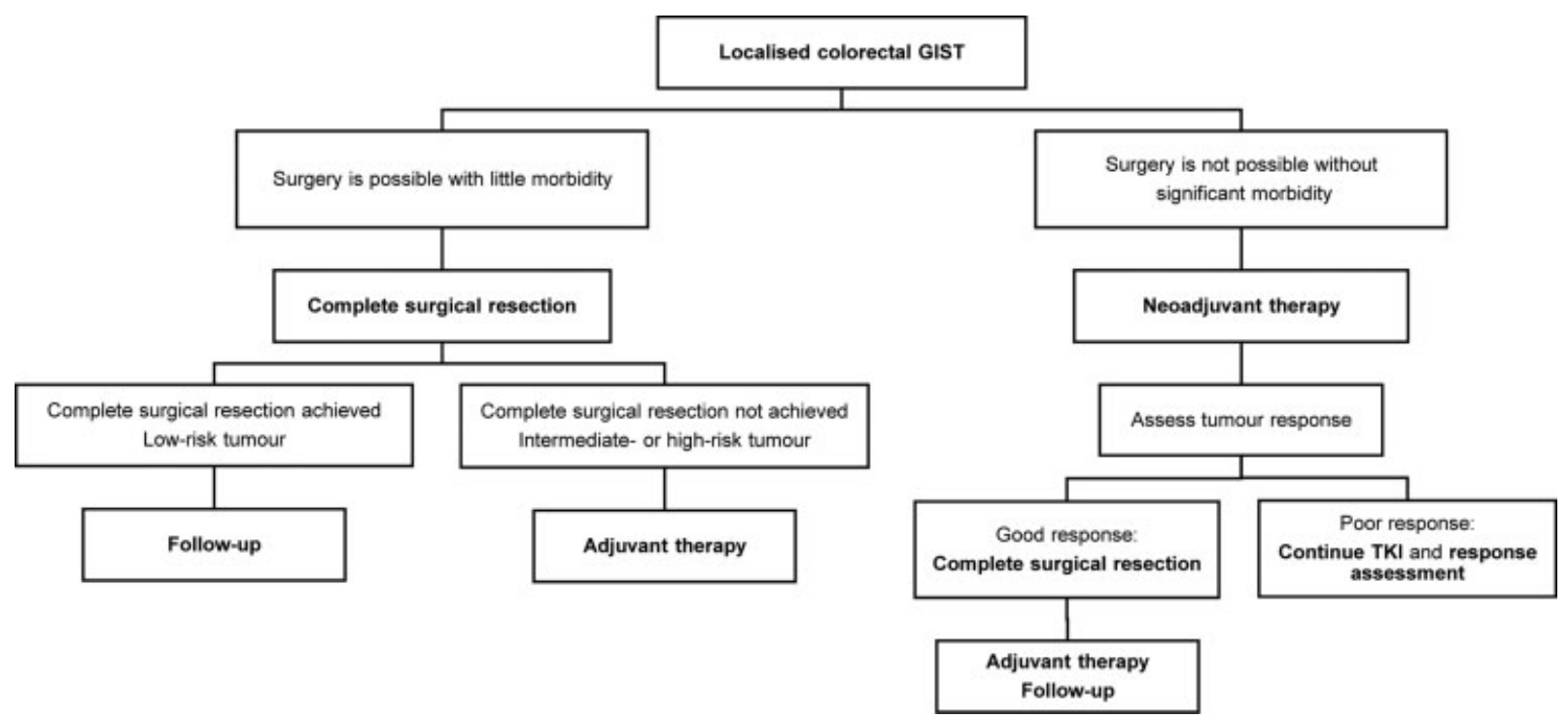

Fig. 1 Algorithm for the approach of localized colorectal gastrointestinal stromal tumors; GIST - gastrointestinal stromal tumor; TKI - tyrosine kinase inhibitor. 


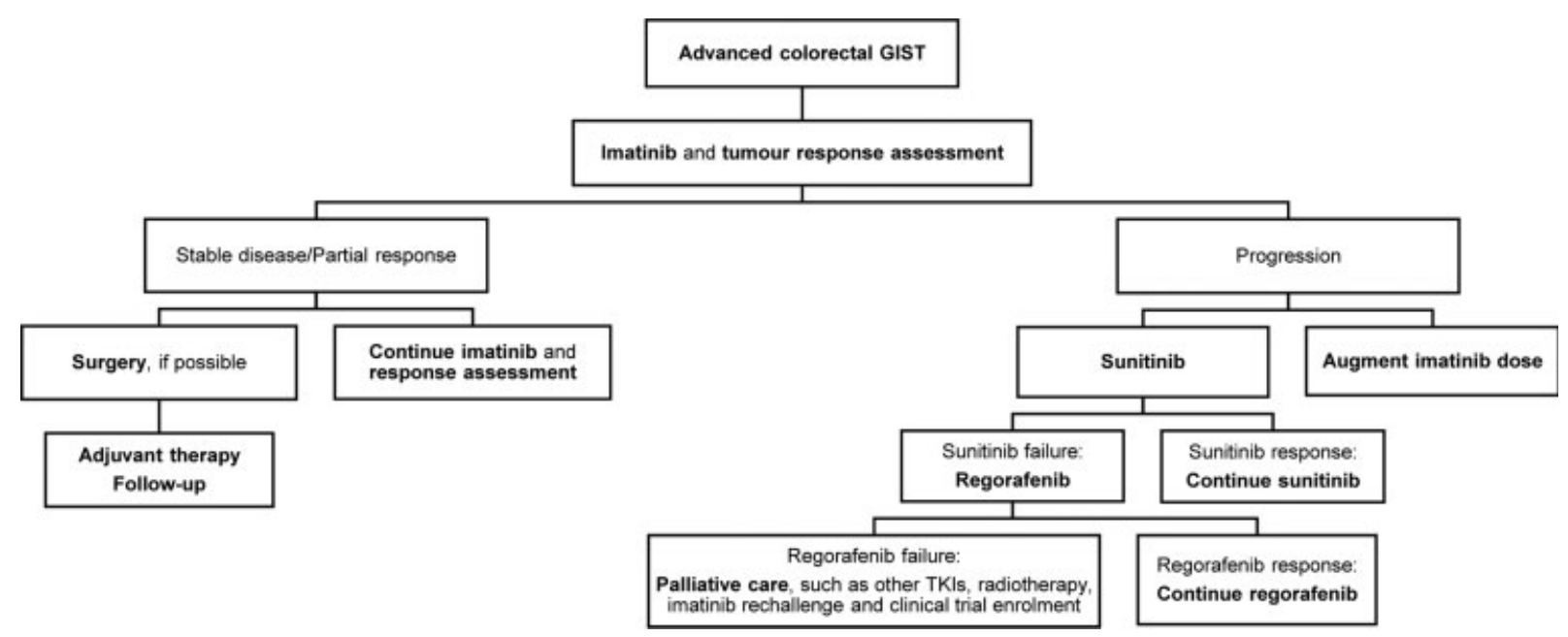

Fig. 2 Algorithm for the approach of advanced colorectal gastrointestinal stromal tumors; GIST - gastrointestinal stromal tumor; TKIs - tyrosine kinase inhibitors.

Hence, in total 60 articles were included in this literature review.

\section{Results}

\section{Prognostic Factors}

Various predictors of colorectal GIST prognosis have been identified and their recognition is paramount to better stratify and manage GIST patients.

For a clearer understanding of this topic, the predictors were grouped into three categories: pathological, immunohistochemical and clinical prognostic factors.

\section{Pathological Prognostic Factors}

The location of the GIST is a determinant of prognosis, highlighted in multiple studies. Wu et al. ${ }^{20}$ reported that the colorectal location was associated with risk of higher recurrence, when compared with the gastric GISTs. Additionally, a study published in $2018^{14}$ found that patients with colorectal GISTs had a survival rate lower by 5 -years when compared with those in gastric and small intestine locations. Moreover, Liu et al. ${ }^{21}$ compared colonic and non-colonic GISTs and reported lower overall survival (OS) $(p=0)$ and cancer-specific survival (CSS) $(p=0.002)$ in colonic GISTs. Kukar et al. ${ }^{22}$ compared gastric with non-gastric GISTs (such as colorectal) and reported a significantly higher disease-specific mortality in the colonic GISTs than in their gastric counterparts (33\% vs $17.5 \% ; p<0.001$ ). Therefore, colon location was an independent factor associated with lower OS (HR [Hazard Ratio $=2.24 ; 95 \%$ confidence interval $[\mathrm{Cl}]$ 1.16-4.31; $p=0.016$ ). Other authors have also reported worse prognosis in colorectal GISTs. ${ }^{5,10,17}$ However, Kukar et al. ${ }^{22}$ did not find statistically significant difference in disease-specific mortality between colonic and rectal GISTs ( $\mathrm{HR}=1.80 ; 95 \% \mathrm{CI} 0.64-5.06$; $p=0.262$ ).

Various studies underline the importance of the mitotic index (cut-off: 5 mitosis/50 HPFs) and tumor size (cut-offs: 2 , 5 and $10 \mathrm{~cm}$ ) in evaluating the GIST's biological activity, insofar they are considered to be the strongest pathological predictors of malignant behavior. ${ }^{18}$ In fact, a study by Tsai et al. ${ }^{23}$ reported the mitotic index as an independent prognostic factor for rectal GISTs (HR $=24.7 ; 95 \% \mathrm{CI} 3.27-186.5$; $p=0.002$ ). Additionally, a multivariate analysis of colonic GISTs by Feng et $a .^{5}$ reported the mitotic index as the only independent prognostic factor for disease-specific survival (DSS) (HR $=7.46$; 95\% CI 1.88-29.63; $p=0.004)$ and diseasefree survival (DFS) $(\mathrm{HR}=4.17 ; 95 \% \mathrm{CI} 1.27-13.72 ; p=0.019)$. A study published in $2018^{10}$ identified tumor size $>5 \mathrm{~cm}$ $(\mathrm{HR}=2.795 ; 95 \%$ CI $1.515-5.157 ; p=0.001)$ and mitotic index $>5 / 50$ HPF $\quad(\mathrm{HR}=3.024 ; 95 \%$ CI $1.856-4.928$; $p<0.001$ ) as the two predictors of lower OS in GIST patients. Despite this, some authors did not find any connection between tumor size or mitotic index and negative prognosis. ${ }^{8,14}$

A higher grade in the Miettinen risk score has also been associated with lower survival rates..$^{5,14,24,25}$ In fact, in a retrospective study conducted by Sakin et al., ${ }^{8}$ the KaplanMeier analysis showed a significantly lower DFS $(p=0.034)$ and OS ( $p=0.022)$ in high-risk patients, compared with their non-high-risk counterparts. Moreover, a study published in $2019^{8}$ reported that high-risk score negatively affected DFS $(\mathrm{HR}=5.158 ; 95 \%$ CI 1.418-12.750; $p=0.013)$.

Lastly, some histological findings such as nuclear pleomorphism, coagulative necrosis and mucosal invasion, can also be useful in determining the prognosis of colorectal GISTs. ${ }^{18,26}$

\section{Immunohistochemical Prognostic Factors}

Beyond morphological factors, various immunohistochemical markers have been shown to be good predictors of patients' prognosis.

One such marker is c-Kit positivity, which has been associated with favorable GIST outcome. A recent study ${ }^{8}$ reported that c-Kit positivity was a positive predictor of DFS, insofar higher levels of this marker were associated with a better prognosis ( $\mathrm{HR}=0.239 ; 95 \%$ CI $0.125-0.689$; $p=0.001$ ). Corroborating this, a different study ${ }^{14}$ also identified c-Kit as a good prognostic predictor (95\% CI 0.979- 
$0.997 ; p=0.012$ ). Nonetheless, a study published in $2018^{10}$ did not report this connection.

The tumor cell proliferation-marker $\mathrm{K}_{\mathrm{i}}-67$ can also be a useful prognostic factor in colorectal GIST, as an association between increased $\mathrm{K}_{\mathrm{i}}-67$ index and worse prognosis has been reported. ${ }^{14,27}$ However, other authors did not find this association. ${ }^{8}$

Although the former immunohistochemical factors are important to predict the colorectal GIST's prognosis, some controversy in the literature still exists. Therefore, other markers have been studied as potential prognostic factors. In fact, a correlation between $\mathrm{p} 53$ and $\mathrm{p} 21$ proteins and other well studied prognostic factors was found in rectal GIST patients, suggesting that high levels of these two markers might be correlated with worse prognosis. ${ }^{26}$ Additionally, a Kaplan-Meier analysis showed that patients with higher p53 and p21 levels had shorter DSS ( $p=0.005, p=0.009$, respectively) than their counterparts. Other markers have recently been associated with good discriminatory capability, such as the hepatoma-derived growth factor and angiogenic markers like CD150, CD31, and VEGF. 27,28

Most of the prognostic factors mentioned so far require tumor-specimen analysis, which may involve invasive and expensive procedures. To overcome this, new prognostic markers have been studied. Stotz et al. ${ }^{29}$ reported a significant relationship between the hemoglobin levels and worse prognosis: decreased recurrence-free survival (RFS) (HR $=0.87 ; 95 \% \mathrm{CI} 0.76-0.99 ; p=0.032)$, and $\mathrm{OS}(\mathrm{HR}=0.86$; $95 \%$ CI 0.74-0.99; $p=0.035$ ). In 2020, a retrospective study ${ }^{13}$ investigating the use of fibrinogen to albumin ratio (FAR) in GIST prognosis reported that FAR was an independent prognostic factor for RFS (HR=3.093; 95\% CI 1.303-7.339; $p=0.01$ ). Other parameters such as the CC chemokine receptor 8 and prognostic nutritional index have also been linked with GIST prognosis. ${ }^{30,31}$

\section{Clinical Prognostic Factors}

The patients' clinical features and treatment received can also influence their prognosis and the overall treatment outcome.

According to most reports in the literature, older age is considered a poor prognostic factor. ${ }^{10,14,24,32,33}$ Additionally, male gender, ${ }^{9,34}$ hematochezia at diagnosis, and tumor rupture during surgery ${ }^{9,34,35}$ are also clinical predictors of prognosis.

This topic will be discussed more in depth later in this paper.

\section{Management of Localized Colorectal GIST}

Surgery remains the treatment of choice for primary localized colorectal GISTs. Since most of these tumors are resectable at presentation, local excision is frequently the surgical procedure of choice, mainly due to two morphologic aspects of these tumors. ${ }^{36}$ First, because the GISTs show an expansive growth pattern, rather than an infiltrative one, so a wide tumor resection is usually not necessary. ${ }^{17,37}$ Second, because lymphatic spread is rare, therefore lymphadenectomy is not necessary in most GISTs. ${ }^{38,39}$ In fact, Li et al. ${ }^{40}$ studied the association between lymphadenectomy and survival in GIST patients and reported lower OS ( $\mathrm{HR}=1.25$; 95\% CI 1.06$11.47 ; p=0.008)$ and CSS (HR $=1.32 ; 95 \%$ CI 1.07-1.64; $p=0.010$ ) in patients who underwent this surgical procedure. Nevertheless, some authors encourage assessing the need for lymphadenectomy in every patient, since it can be required in certain situations, such as in case of enlarged lymph nodes. ${ }^{40}$

During surgery it is important to guarantee complete tumor resection and avoid tumor rupture. In fact, incomplete tumor removal is reserved to palliate symptoms, such as hemorrhage. ${ }^{39}$ A 2018 cohort study ${ }^{10}$ reported that curative resection was a positive predictor of OS ( $\mathrm{HR}=3.384 ; 95 \% \mathrm{CI}$ $1.729-6.621 ; p<0.001)$. Moreover, Shu et al. ${ }^{25}$ reported that microscopically positive margins led to worse RFS (HR $=4.207 ; 95 \%$ CI 1.340-13.214; $p=0.014$ ). However, in a study conducted by Jakob et al. ${ }^{41}$ tumor resection with negative margins was not associated with increased DFS $(p=0.10)$ nor OS $(p=0.27)$.

Since they have unique characteristics and different surgical approaches, the colonic and rectal GISTs should be managed separately. ${ }^{22} \mathrm{~A}$ segmental colectomy is usually the procedure of choice for colonic GISTs. ${ }^{37}$ The surgery of rectal GISTs is not as straightforward and can be challenging, mostly because it can be difficult to preserve anal sphincter, and because surgery in the pelvis can be technically difficult. ${ }^{42}$

The surgical approaches of rectal GISTs can be grouped in two categories: local resection and radical excision. ${ }^{33,39}$ The former includes techniques such as transanal excision, transanal endoscopic microsurgery (TEM) and transanal minimally invasive surgery (TAMIS), while the latter includes low anterior resection, abdominoperineal resection, pelvic exenteration, and intersphincteric resection (ISR), amongst others. ${ }^{16,33,38,39,43}$ Even though some authors defend excising small rectal GISTs by endoscopic resection, the endoscopic treatment for rectal GISTs remains controversial. ${ }^{25}$

When compared with more extensive techniques, local resection through transanal surgery is associated with lower surgical trauma, higher anus-sphincter preservation, faster recovery, and overall lower complication rates and morbidity. ${ }^{39,44}$

However, local resection might not be the appropriate surgical procedure for all rectal GISTs, and sometimes extensive surgical approaches are required. ${ }^{4,38,43}$ Large and aggressive rectal GISTs with extensive extra-rectal growthinvading adjacent structures, such as the prostate, vagina, or sacrum-usually require a more extensive resection. ${ }^{9,45,46}$

A study published in $2020^{39}$ did not find statistically significant differences in anus preservation $(p=0.100)$, combined organ resection ( $p=0.428), 5$-year DFS ( $p=0.952$ ) and OS $(p=0.832)$ between a group of rectal GIST patients who underwent local resection and a group who underwent radical excision. A different study ${ }^{44}$ supported these results, insofar the authors did not find significant differences in DFS $(p=0.243)$ nor in OS $(p=0.308)$ between transanal and nontransanal approaches. Additionally, a study published by IJzerman et al. ${ }^{32}$ did not report a difference in RFS 
between local resection and radical excision surgeries $(p=0.782)$. Nonetheless, a study conducted by Shu et al. ${ }^{25}$ reported a significantly lower rate of R0 resection $(p=0.015)$ and longer postoperative hospital stay $(p=0.004)$ in the segmental resection group than in the local excision group. Even though the choice of surgical method did not affect RFS $(p=0.823)$, patients in the local excision group had better OS $(p=0.049)$.

Since complete tumor removal may implicate considerable morbidity, and because disease recurrence is highly prevalent, perioperative medical therapy has gained importance in the management of colorectal GIST. ${ }^{19}$

In fact, the discovery of GIST's molecular pathophysiology, led to the introduction of tyrosine kinase inhibitors (TKIs), which have revolutionized the management of this disease. Imatinib, sunitinib and regorafenib are, respectively, the first-, second- and third-line TKIs used in GIST treatment. Unfortunately, not all GISTs show the same sensitivity toTKIs, and some might not even respond to these drugs. Thus, it is important to predict GIST response to a certain TKI through molecular analysis, to assess which patients will benefit from this therapy. ${ }^{42}$ The association between the GIST's mutational status and TKI response is beyond the scope of this paper and can be found elsewhere. ${ }^{7,12,42}$ Furthermore, it is now known that location has an impact in tumor response to TKIs, since non-gastric GISTs have worse response to these drugs than their gastric counterparts. ${ }^{15}$

The patients with localized diseases in whom complete surgical resection may not be possible without considerable morbidity are the ones who benefit the most from neoadjuvant TKIs (-Fig. $\mathbf{1}$ ). In fact, it is now known that TKIs before surgery can be of extreme importance in colorectal GISTs. They minimize the risk of locoregional recurrence and intraperitoneal dissemination by reducing the risk of pseudocapsule rupture during surgery, by enabling a more complete tumor resection, and by promoting loss of tumor vascularity. Additionally, TKIs can minimize surgical complications, allow less invasive procedures, and, by reducing tumor bulk, they facilitate surgical resection in complicated areas, such as the lower rectum. However, complete response to TKIs is rare..$^{4,9,16,24,42,47}$ During neoadjuvant therapy it is necessary to assess the patient's adhesion to therapy, as well as assess tumor response through imageology (-Fig. 1). ${ }^{48,49}$

Wilkinson et al. ${ }^{9}$ reported a significant reduction in median tumor size $(p<0.001)$ and mitotic count $(p=0.015)$ in patients receiving neoadjuvant imatinib. Additionally, 7 out of 9 patients assessed to need anal sphincter sacrifice underwent sphincter-preserving surgery after use of neoadjuvant imatinib. A study conducted by Zanwar et al. ${ }^{50}$ estimated that one third of the rectal GIST patients would have had sphincter-sacrificing surgery if they had not received imatinib before the operation.

Nevertheless, some drawbacks to neoadjuvant imatinib have been described. A study published by Lee et al. ${ }^{51}$ reported a higher rate of complications in patients receiving neoadjuvant imatinib, including higher anastomosis insufficiency, suggesting that TKI therapy can compromise anastomotic healing.
Beyond its role in surgery, neoadjuvant imatinib also has an impact in patients' prognosis. In a study conducted by Hawkins et al., ${ }^{33}$ patients who received neoadjuvant imatinib had better OS than patients who did not $(p=0.03)$. Additionally, Cavnar et al. ${ }^{4}$ proved that high-risk patients who had received any type of perioperative imatinib therapy had longer OS compared with those who did not ( $91 \%$ vs $47 \%, p=0.049$ ). Another example is the study performed by Jakob et al., ${ }^{41}$ which reported a significant increase in OS $(p=0.03)$ and DFS $(p<0.01)$ in rectal GIST patients receiving perioperative imatinib. Furthermore, Zanwar et al. ${ }^{50}$ reported statistically improved DFS in a group of patients receiving neoadjuvant imatinib when compared with those who did not (70 vs 120 months; $p=0.039$ ). Despite these positive results, a recent study by Shu et al. ${ }^{25}$ did not discover OS benefit in patients receiving neoadjuvant imatinib ( $p=0.355)$.

As evidenced here, there are numerous advantages to preoperative TKIs. However, the duration of treatment remains controversial. ${ }^{43,47}$ The challenge lies in the perfect timing for surgery, as long preoperative treatment may allow tumor progression and short treatment may not enable maximal tumor downsizing. ${ }^{42,43,47}$ Various studies have been published focusing on this topic. A retrospective study by Tang et al. ${ }^{47}$ reported greater tumor downsizing after 6 to 12 months of TKI onset, with most tumors stabilizing their size at 12 months. A prospective study published in $2019^{36}$ reported a median time of 6.9 months for maximal rectal GIST downsizing in patients with locally advanced disease. Despite these results, a different study ${ }^{50}$ did not find any significant difference in sphincter preservation rate between patients receiving less or more than 12 months of neoadjuvant imatinib.

Even after neoadjuvant therapy and surgery, some colorectal GIST patients still have significant risk of disease relapse. Therefore, imatinib use was studied in the adjuvant setting, with the goal of minimizing the recurrence of colorectal GIST ( - Fig. 1). Dematteo et al. ${ }^{52}$ reported a longer RFS in patients receiving adjuvant imatinib $(\mathrm{HR}=0.35 ; 95 \%$ CI $0.22-0.53 ; p<0.0001)$. Similarly, a study published in $2015^{6}$ reported an improved RFS (HR $=0.23$; 95\% CI $0.07-$ $0.80 ; p=0.012)$ and $\mathrm{OS}(\mathrm{HR}=0.20 ; 95 \%$ CI $0.05-0.91$; $p=0.021)$ in colorectal GIST patients receiving imatinib after surgery. Different studies have also reported an association between adjuvant therapy, longer DFS ( $\mathrm{HR}=0.365 ; 95 \% \mathrm{CI}$ $0.199-0.918 ; p=0.023),{ }^{8}$ and longer OS $(\mathrm{HR}=0.262 ; 95 \% \mathrm{CI}$ $0.122-0.563 ; p=0.001){ }^{10}$

Beyond minimizing disease recurrence, adjuvant therapy can also be useful in other situations, such as delaying tumor recurrence in case of intraoperative tumor rupture, ${ }^{53}$ and avoiding further surgical resections in patients with incomplete tumor removal (-Fig. 1).$^{4,9}$

As for neoadjuvant therapy, there is still controversy regarding treatment duration of adjuvant TKIs. To clarify this, Joensuu et al. ${ }^{54}$ compared adjuvant therapy of one- and threeyear-long duration. The authors discovered more benefits from three years of adjuvant imatinib use, as it was associated with longer $\mathrm{RFS}(\mathrm{HR}=0.46 ; 95 \% \mathrm{CI} 0.32-0.65 ; p<0.001)$ and longer OS $(\mathrm{HR}=0.45 ; 95 \%$ CI $0.22-0.89 ; p=0.02)$. 


\section{Management of Advanced Colorectal GIST}

Advanced disease encompasses unresectable and metastatic disease. It represents the most frequent cause of treatment failure in colorectal GISTs and is associated with poor prognosis. $^{5}$

The colorectal GISTs usually metastasize to the liver and peritoneum, with metastases to the latter being associated with poorer prognosis. ${ }^{53}$ The rectal GISTs can also metastasize to the lung and bone, ${ }^{8,19}$ even though they usually recur in a locoregional fashion. $24,38,42$

Imatinib is the first-line treatment option for advanced disease patients with sensitive mutations to this TKI. ${ }^{12}$ It is important to assess patient adhesion to therapy, as well as tumor response through imageology, as some tumors might progress while on imatinib (-Fig. 2). ${ }^{49}$

According to the European Society for Medical Oncology (ESMO) guidelines, ${ }^{48}$ imatinib should be continued for an unlimited period of time in the setting of advanced disease since its discontinuation can be associated with tumor progression.

Despite this, most patients develop imatinib resistance within a few years after treatment onset through secondary mutations in KIT or PDGFR $\alpha$ oncogenes. ${ }^{12,55,56}$ After imatinib failure, it is plausible to either switch to sunitinib, the second-line approved TKI for advanced disease, or escalate imatinib dose (-Fig. 2). ${ }^{12,19}$

Due to its different mechanism of action, regorafenib has been approved as a third-line therapy for GISTs that do not respond to imatinib and sunitinib (-Fig. 2). ${ }^{56}$ A metaanalysis published in $2017^{55}$ reported clinical benefit in 49\% (95\% CI 30-67\%) and partial tumor response in $14 \%$ (95\% CI 5-23\%) cases of advanced GISTs being treated with regorafenib as a third-line TKI option. Additionally, the GRID trial $^{57}$ showed improvement in progression-free survival (PFS) in advanced GIST patients receiving regorafenib, compared with those who did not (4.8 vs 0.9 months; $H R=0.27$; 95\% CI 0.19-0.39; $p<0.0001)$. However, no improvement in OS was reported (HR 0.77, 95\% CI 0.42-1.41; $p=0.199$ ).

Surgery also plays a role in case of advanced disease. Since tumor volume is an important determinant of tumor resistance to imatinib, reducing tumor bulk through cytoreductive surgery may improve GIST patients' prognosis and improve TKIs' effectiveness. ${ }^{58-60}$ It has been suggested that the patients who benefit the most from cytoreductive surgery are the ones who respond to TKI therapy, those who have few metastatic foci, and those in whom complete surgical resection is possible (-Fig. 2) ${ }^{2,58}$ Conversely, surgery has little benefit before imatinib initiation and in patients with generalized disease progression. ${ }^{58}$ Therefore, most authors recommend operating patients between 6 months and 2 years after TKI onset, to confirm stable disease or partial response and to avoid the accumulation of new mutations. ${ }^{58}$

Finally, the GIST may progress even after first-, second-, and third-line TKIs. In this scenario, little is known on how to proceed. The National Comprehensive Cancer Network (NCCN) guidelines suggest enrolling the patient on a clinical trial or providing the best supportive care, such as reintro- duction of a previously tolerated TKI, to palliate symptoms (-Fig. 2). ${ }^{49}$

\section{Follow-up}

As previously mentioned, colorectal GISTs have high risk of recurrence and progression even after surgery. Therefore, long term follow-up is usually warranted. ${ }^{18,19}$

Despite the lack of standard recommendations for GIST follow-up, the NCCN guidelines suggest surveillance history, physical examination and abdominopelvic CT scan every 3 to 6 months in the first 3 to 5 years after surgery, and annually thereafter. $^{49}$

However, follow-up should be tailored to each patient, since the small GISTs $(<2 \mathrm{~cm})$ with low mitotic activity can have a less frequent surveillance, while high-risk GISTs may require closer follow-up. ${ }^{48,49}$

\section{Discussion}

Even though all GISTs have malignant potential, not all of them will become malignant. Therefore, it is important to identify prognostic factors since they allow a better prediction of tumor malignancy, enabling a more precise tumor approach. ${ }^{5,9}$ They can discriminate patients, identifying those who only require surgery, and those in whom medical therapy might also be necessary.

Since the GIST is a heterogeneous disease along the GI tract, differences in predictors of malignancy between tumors at different locations exist. As a matter of fact, it is now known that colorectal GISTs have a poorer prognosis and higher risk of recurrence than GISTs in other locations. Therefore, these tumors might require longer therapy and closer follow-up. ${ }^{15}$

Despite this, controversy amongst authors still exists. In fact, even though immunohistochemistry is a universally acceptable tool for GIST diagnosis, its use as a prognosis predictor divides the authors. Two examples are c-Kit positivity and high $\mathrm{K}_{\mathrm{i}}-67$ index. While some authors describe a relationship between these markers and prognosis, others do not report this association. $8,10,14,27$

To overcome these divergences and improve prognosis determination with better patient stratification, new determinants have recently been studied, showing promising results. Moreover, some of these parameters, such as angiogenic markers, are also being studied as new therapeutic targets.

A standard treatment approach for colorectal GIST is also lacking. Therefore, it is essential that therapy is decided by a multidisciplinary team in a specialized center, to provide the best oncological outcomes while avoiding serious comorbidities.

Surgery is the only potentially curative therapy. However, different patients require different surgical approaches. Therefore, the choice of surgical procedure should depend on the tumor's characteristics, as well as the surgeon's opinion and patient's choice.

There isn't an agreement between choosing local resection and radical excision for rectal GISTs. Even though Gou et al. $^{39}$ did not find differences in OS between patients undergoing local resection and radical excision, Shu et al. ${ }^{25}$ 
reported a better OS in the local excision group. Despite this disagreement, surgeries where local resection is a possibility are preferred, as they allow good tumor removal with little morbidity, reducing the need to sacrifice the anal sphincter and the need for permanent colostomies.

The introduction of TKIs in the localized and advanced disease settings improved GIST management and patient's prognosis. Since not all tumors show the same sensitivity to TKIs, the mutational analysis became part of routine GIST management.

In our review it was consistent that the use of neoadjuvant imatinib reduces peri-operative complications and allows for less extensive surgeries. Additionally, the benefit of imatinib use before surgery in improving patients' RFS and OS was supported by most authors, with the exception of Shu et al., ${ }^{25}$ as they did not report this relationship.

Despite the clear benefits of neoadjuvant imatinib, controversy regarding the timing of surgery still exists. Even though most authors defend operating patients after maximal TKI response and before tumor progression, the exact treatment duration varies between studies. ${ }^{19,42,43,47}$ Therefore, it is important to evaluate tumor progression through imageology and discuss the timing of surgery after imatinib use in a multidisciplinary team. ${ }^{19}$

Few studies in the literature focus on the duration of imatinib use after surgery. The most accepted study regarding high-risk patients is the paper by Joensuu H. et al., ${ }^{54}$ where they defend treatment should last for three years. For intermediate-risk GISTs, Wu et al. ${ }^{20}$ suggest duration of one year of postoperative imatinib use. For low-risk patients, the ESMO and NCCN guidelines recommend patient follow-up, without the need for adjuvant imatinib, as the risk of recurrence is low. ${ }^{48,49}$

Imatinib remains the first-line of treatment for unresectable and metastatic GISTs. Sunitinib and regorafenib can be used in case of disease progression while on imatinib. However, some tumors might not respond to these TKIs, or even become resistant to them. In these situations, palliative care should be offered to patients.

Since there isn't a consensus regarding the colorectal GIST follow-up, it should be personalized according to recurrence risk assessment.

Lastly, this review has some limitations that are worth noticing. The colorectal GISTs are exceedingly rare and most studies in the literature are case-reports or studies comprising few patients. On top of that, most studies combine GISTs of all sites, not focusing only on the colorectal location. This may contribute to the still existing controversy and lack of studies regarding management and prognosis of these tumors. Another limitation of our review concerns the variability of inclusion and exclusion criteria of the studies used. For example, some authors excluded patients with metastatic disease while others only included patients with high-risk GISTs. Furthermore, the use of different prognostic parameters between studies might also contribute to the differences in the results obtained. Hence, caution should be taken when extrapolating the results, as they might not apply to all colorectal GIST patients.
To overcome these limitations and reduce controversy between studies, larger studies, with more patients and more prospective studies are needed in the future. Thereafter, it might be possible to gather more evidence, which will allow for the definition of a standard colorectal GIST management approach and to find better prognostic determinants to be included in the current prognostic scores.

\section{Conclusion}

The colorectal GIST is a rare tumor with malignant potential and high risk of recurrence.

Various prognostic factors have already been identified, and the role of surgery and medical therapy has become clearer throughout the years. However, controversy regarding the management and prognosis of this tumor still exists. Therefore, personalized management must be the norm, and each case should be analyzed in the setting of a multidisciplinary team.

Future studies are needed to clarify the discrepancies in the literature and describe standard management approaches for colorectal GIST patients, maximizing their oncological outcomes.

\section{Sources of Support of Study Development \\ None}

Conflict of Interests

The authors have no conflict of interests to declare.

\section{References}

1 Nishida T, Goto O, Raut CP, Yahagi N. Diagnostic and treatment strategy for small gastrointestinal stromal tumors. Cancer 2016; 122(20):3110-3118. Doi: 10.1002/cncr.30239

2 Gaitanidis A, El Lakis M, Alevizakos M, Tsaroucha A, Pitiakoudis M. Predictors of lymph node metastasis in patients with gastrointestinal stromal tumors (GISTs). Langenbecks Arch Surg 2018;403 (05):599-606. Doi: 10.1007/s00423-018-1683-0

3 Porter MG, Stoeger SM. Atypical Colorectal Neoplasms. Surg Clin North Am 2017;97(03):641-656. Doi: 10.1016/j.suc.2017.01.011

4 Cavnar MJ, Wang L, Balachandran VP, et al. Rectal Gastrointestinal Stromal Tumor (GIST) in the Era of Imatinib: Organ Preservation and Improved Oncologic Outcome. Ann Surg Oncol 2017;24(13): 3972-3980. Doi: 10.1245/s10434-017-6087-9

5 Feng F, Tian Y, Liu Z, et al. Clinicopathological features and prognosis of colonic gastrointestinal stromal tumors: evaluation of a pooled case series. Oncotarget 2016;7(26):40735-40745. Doi: 10.18632/oncotarget.9196

$6 \mathrm{Li}$ Y, Meng X. The efficacy of adjuvant imatinib therapy in improving the prognosis of patients with colorectal gastrointestinal stromal tumours. Ann R Coll Surg Engl 2015;97(03): 215-220. Doi: $10.1308 / 003588414 \times 14055925061432$

7 Kang W, Zhu C, Yu J, Ye X, Ma Z. KIT gene mutations in gastrointestinal stromal tumor. Front Biosci 2015;20:919-926. Doi: $10.2741 / 4346$

8 Sakin A, Can O, Arici S, et al. Factors Affecting Disease-Free Survival in Operated Nonmetastatic Gastrointestinal Stromal Tumors. J Surg Res 2019;241:170-177. Doi: 10.1016/j. jss.2019.03.059

9 Wilkinson MJ, Fitzgerald JE, Strauss DC, et al. Surgical treatment of gastrointestinal stromal tumour of the rectum in the era of imatinib. Br J Surg 2015;102(08):965-971. Doi: 10.1002/bjs.9818 
10 Liu X, Qiu H, Zhang P, et al; China Gastrointestinal Stromal Tumor Study Group (CN-GIST) Prognostic factors of primary gastrointestinal stromal tumors: a cohort study based on high-volume centers. Chin J Cancer Res 2018;30(01):61-71. Doi: 10.21147/j. issn.1000-9604.2018.01.07

11 Kelley KA, Byrne R, Lu KC. Gastrointestinal Stromal Tumors of the Distal Gastrointestinal Tract. Clin Colon Rectal Surg 2018;31(05): 295-300. Doi: 10.1055/s-0038-1642053

12 Blay JY, Shen L, Kang YK, et al. Nilotinib versus imatinib as firstline therapy for patients with unresectable or metastatic gastrointestinal stromal tumours (ENESTg1): a randomised phase 3 trial. Lancet Oncol 2015;16(05):550-560. Doi: 10.1016/s14702045(15)70105-1

13 Li R, Song S, He X, et al. Relationship Between Fibrinogen to Albumin Ratio and Prognosis of Gastrointestinal Stromal Tumors: A Retrospective Cohort Study. Cancer Manag Res 2020; 12:8643-8651. Doi: 10.2147/cmar.S271171

14 Hatipoğlu E, Demiryas S. Gastrointestinal stromal tumors: 16 years' experience within a university hospital. Rev Esp Enferm Dig 2018;110(06):358-364. Doi: 10.17235/reed.2018.5199/2017

15 Qi J, Liu HL, Ren F, et al. Preoperative adjuvant therapy for locally advanced and recurrent/metastatic gastrointestinal stromal tumors: a retrospective study. World J Surg Oncol 2020;18(01): 70. Doi: 10.1186/s12957-020-01840-9

16 Han X, Xu J, Qiu H, Lin G. A Novel Curative Treatment Strategy for Patients with Lower Grade Rectal Gastrointestinal Stromal Tumor: Chemoreduction Combined with Transanal Endoscopic Microsurgery. J Laparoendosc Adv Surg Tech A 2017;27(06): 579-585. Doi: 10.1089/lap.2017.0051

17 Almaazmi H, Stem M, Lo BD, et al. The Impact of Imatinib on Survival and Treatment Trends for Small Bowel and Colorectal Gastrointestinal Stromal Tumors. J Gastrointest Surg 2020;24 (01):98-108. Doi: 10.1007/s11605-019-04344-4

18 Miettinen M, Lasota J. Gastrointestinal stromal tumors: pathology and prognosis at different sites. Semin Diagn Pathol 2006;23(02): 70-83. Doi: 10.1053/j.semdp.2006.09.001

19 Theodoropoulos DG. Gastrointestinal tumors of the colon and rectum. Clin Colon Rectal Surg 2011;24(03):161-170. Doi: 10.1055/s-0031-1286000

20 Wu X, Li J, Xu W, Gao J, Li Y, Shen L. Postoperative imatinib in patients with intermediate risk gastrointestinal stromal tumor. Future Oncol 2018;14(17):1721-1729. Doi: 10.2217/fon-2017-0691

21 Liu Z, Sun Y, Li Y, et al. Colonic Gastrointestinal Stromal Tumor: A Population-Based Analysis of Incidence and Survival. Gastroenterol Res Pract 2019;2019:3849850. Doi: 10.1155/2019/3849850

22 Kukar M, Kapil A, Papenfuss W, Groman A, Grobmyer SR, Hochwald SN. Gastrointestinal stromal tumors (GISTs) at uncommon locations: a large population based analysis. J Surg Oncol 2015; 111(06):696-701. Doi: 10.1002/jso.23873

23 Tsai MC, Lin JW, Lin SE, Chen HH, Lee CM, Hu TH. Prognostic analysis of rectal stromal tumors by reference of National Institutes of Health risk categories and immunohistochemical studies. Dis Colon Rectum 2008;51(10):1535-1543. Doi: 10.1007/ s10350-008-9370-9

24 Stuart E, Banerjee S, de la Torre J, et al. Frequent rectal gastrointestinal stromal tumor recurrences in the imatinib era: Retrospective analysis of an International Patient Registry. J Surg Oncol 2019;120(04):715-721. Doi: 10.1002/jso.25621

25 Shu P, Sun XF, Fang Y, et al. Clinical outcomes of different therapeutic modalities for rectal gastrointestinal stromal tumor: Summary of 14-year clinical experience in a single center. Int J Surg 2020;77:1-7. Doi: 10.1016/j.ijsu.2020.03.007

$26 \mathrm{Hu} \mathrm{TH}$, Tai MH, Chuah SK, et al. Elevated p21 expression is associated with poor prognosis of rectal stromal tumors after resection. J Surg Oncol 2008;98(02):117-123. Doi: 10.1002/ jso. 21094

27 Basilio-de-Oliveira RP, Pannain VL. Prognostic angiogenic markers (endoglin, VEGF, CD31) and tumor cell proliferation
(Ki67) for gastrointestinal stromal tumors. World J Gastroenterol 2015;21(22):6924-6930. Doi: 10.3748/wjg.v21.i22.6924

$28 \mathrm{Hu}$ TH, Lin JW, Chen HH, Liu LF, Chuah SK, Tai MH. The expression and prognostic role of hepatoma-derived growth factor in colorectal stromal tumors. Dis Colon Rectum 2009;52(02):319-326. Doi: 10.1007/DCR.0b013e31819d1666

29 Stotz M, Liegl-Atzwanger B, Posch F, et al. Blood-Based Biomarkers Are Associated with Disease Recurrence and Survival in Gastrointestinal Stroma Tumor Patients after Surgical Resection. PLoS One 2016;11(07):e0159448. Doi: 10.1371/journal. pone. 0159448

$30 \mathrm{Li} \mathrm{HL}$, Wang LH, Hu YL, et al. Clinical and prognostic significance of CC chemokine receptor type 8 protein expression in gastrointestinal stromal tumors. World J Gastroenterol 2020;26(31):4656-4668. Doi: 10.3748/wjg.v26.i31.4656

31 Sun J, Mei Y, Zhu Q et al. Relationship of prognostic nutritional index with prognosis of gastrointestinal stromal tumors. J Cancer 2019;10(12):2679-2686. Doi: 10.7150/jca.32299

32 IJzerman NS, Mohammadi M, Tzanis D, et al. Quality of treatment and surgical approach for rectal gastrointestinal stromal tumour (GIST) in a large European cohort. Eur J Surg Oncol 2020;46(06): 1124-1130. Doi: 10.1016/j.ejso.2020.02.033

33 Hawkins AT, Wells KO, Krishnamurty DM, et al. Preoperative Chemotherapy and Survival for Large Anorectal Gastrointestinal Stromal Tumors: A National Analysis of 333 Cases. Ann Surg Oncol 2017;24(05):1195-1201. Doi: 10.1245/s10434-016-5706-1

34 Joensuu H, Vehtari A, Riihimäki J, et al. Risk of recurrence of gastrointestinal stromal tumour after surgery: an analysis of pooled population-based cohorts. Lancet Oncol 2012;13(03): 265-274. Doi: 10.1016/s1470-2045(11)70299-6

35 Xiao CC, Zhang S, Wang MH, et al. Clinicopathological features and prognostic factors of rectal gastrointestinal stromal tumors. J Gastrointest Surg 2013;17(04):793-798. Doi: 10.1007/s11605012-2086-0

36 Wang SY, Wu CE, Lai CC, et al. Prospective Evaluation of Neoadjuvant Imatinib Use in Locally Advanced Gastrointestinal Stromal Tumors: Emphasis on the Optimal Duration of Neoadjuvant Imatinib Use, Safety, and Oncological Outcome. Cancers (Basel) 2019;11(03):E424. Doi: 10.3390/cancers11030424

37 Amato A. Colorectal gastrointestinal stromal tumor. Tech Coloproctol 2010;14(Suppl 1):S91-S95. Doi: 10.1007/s10151-0100631-y

38 Yasui M, Tsujinaka T, Mori M, Takahashi T, Nakashima Y, Nishida TKinki GIST Study Group. Characteristics and prognosis of rectal gastrointestinal stromal tumors: an analysis of registry data. Surg Today 2017;47(10):1188-1194. Doi: 10.1007/s00595-0171524-8

39 Guo W, Yang Z, Wei Y, et al. Radical excision versus local resection for primary rectal gastrointestinal stromal tumors. Cohort Study. Int J Surg 2020;77:190-197. Doi: 10.1016/j.ijsu.2020.03.068

40 Li C, Su D, Xie C, Chen Q, Zhou J, Wu X. Lymphadenectomy is associated with poor survival in patients with gastrointestinal stromal tumors. Ann Transl Med 2019;7(20):558. Doi: 10.21037| atm.2019.09.60

41 Jakob J, Mussi C, Ronellenfitsch U, et al. Gastrointestinal stromal tumor of the rectum: results of surgical and multimodality therapy in the era of imatinib. Ann Surg Oncol 2013;20(02): 586-592. Doi: 10.1245/s10434-012-2647-1

42 Kaneko M, Emoto S, Murono K, et al. Neoadjuvant imatinib therapy in rectal gastrointestinal stromal tumors. Surg Today 2019;49(06):460-466. Doi: 10.1007/s00595-018-1737-5

43 Kaneko M, Nozawa H, Emoto S, et al. Neoadjuvant Imatinib Therapy Followed by Intersphincteric Resection for Low Rectal Gastrointestinal Stromal Tumors. Anticancer Res 2017;37(09): 5155-5160. Doi: 10.21873/anticanres.11936

44 Yang Z, Guo W, Huang R, Hu M, Wang H, Wang H. Transanal versus nontransanal surgery for the treatment of primary rectal gastrointestinal stromal tumors: a 10-year experience in a high-volume 
center. Ann Transl Med 2020;8(05):201. Doi: 10.21037/ atm.2020.01.55

45 Liu H, Yan Z, Liao G, Yin H. Treatment strategy of rectal gastrointestinal stromal tumor (GIST). J Surg Oncol 2014;109(07): 708-713. Doi: $10.1002 /$ jso.23562

46 Gervaz P, Huber O, Morel P. Surgical management of gastrointestinal stromal tumours. Br J Surg 2009;96(06):567-578. Doi: 10.1002/bjs.6601

47 Tang S, Yin Y, Shen C, et al. Preoperative imatinib mesylate (IM) for huge gastrointestinal stromal tumors (GIST). World J Surg Oncol 2017;15(01):79. Doi: 10.1186/s12957-017-1143-2

48 Casali PGAea. Gastrointestinal Stromal Tumours NESMO-EURACAN Clinical Practice Guidelines for diagnosis, treatment and follow-up. Guideline. Ann Oncol 2018;29(04):68-78

49 National Comprehensive Cancer NetworK von Mehren M KJ, et al. NCCN Clinical Practice Guidelines in Oncology: Gastrointestinal Stromal Tumors (GISTs) Version 1.2021. Accessed January 16, 2021, https://www.nccn.org/professionals/physician_gls/default. aspx

50 Zanwar S, Ostwal V, Sahu A, et al. Rectal GIST-Outcomes and viewpoint from a tertiary cancer center. Indian J Gastroenterol 2016;35(06):445-449. Doi: 10.1007/s12664-016-0710-8

51 Lee JC, Chen CH, Chen TC, Yeh CN, Yeh TS. Preoperative tyrosine kinase inhibitors risks bowel anastomotic healing in patients with advanced primary and recurrent/metastatic gastrointestinal stromal tumors-A rose has its thorns. Eur J Surg Oncol 2019;45(02): 153-159. Doi: 10.1016/j.ejso.2018.09.029

52 Dematteo RP, Ballman KV, Antonescu CR, et al; American College of Surgeons Oncology Group (ACOSOG) Intergroup Adjuvant GIST Study Team. Adjuvant imatinib mesylate after resection of localised, primary gastrointestinal stromal tumour: a randomised, double-blind, placebo-controlled trial. Lancet 2009;373 (9669):1097-1104. Doi: 10.1016/s0140-6736(09)60500-6
53 Jakob J, Hohenberger P. Neoadjuvant Therapy to Downstage the Extent of Resection of Gastrointestinal Stromal Tumors. Visc Med 2018;34(05):359-365. Doi: 10.1159/000493405

54 Joensuu H, Eriksson M, Sundby Hall K, et al. One vs three years of adjuvant imatinib for operable gastrointestinal stromal tumor: a randomized trial. JAMA 2012;307(12):1265-1272. Doi: 10.1001/ jama.2012.347

55 Zhang Z, Jiang T, Wang W, Piao D. Efficacy and safety of regorafenib for advanced gastrointestinal stromal tumor after failure with imatinib and sunitinib treatment: A meta-analysis. Medicine (Baltimore) 2017;96(48):e8698. Doi: 10.1097/ md.0000000000008698

56 Grothey A, Blay JY, Pavlakis N, Yoshino T, Bruix J. Evolving role of regorafenib for the treatment of advanced cancers. Cancer Treat Rev 2020;86:101993. Doi: 10.1016/j.ctrv.2020.101993

57 Demetri GD, Reichardt P, Kang YK, et al; GRID study investigators. Efficacy and safety of regorafenib for advanced gastrointestinal stromal tumours after failure of imatinib and sunitinib (GRID): an international, multicentre, randomised, placebo-controlled, phase 3 trial. Lancet 2013;381(9863):295-302. Doi: 10.1016/ s0140-6736(12)61857-1

58 Keung EZ, Fairweather M, Raut CP. The Role of Surgery in Metastatic Gastrointestinal Stromal Tumors. Curr Treat Options Oncol 2016;17(02):8. Doi: 10.1007/s11864-015-0384-y

$59 \mathrm{Du}$ CY, Zhou Y, Song C, et al. Is there a role of surgery in patients with recurrent or metastatic gastrointestinal stromal tumours responding to imatinib: a prospective randomised trial in China. Eur J Cancer 2014;50(10):1772-1778. Doi: 10.1016/j.ejca.2014.03.280

60 Patrikidou A, Chabaud S, Ray-Coquard I, et al; French Sarcoma Group. Influence of imatinib interruption and rechallenge on the residual disease in patients with advanced GIST: results of the BFR14 prospective French Sarcoma Group randomised, phase III trial. Ann Oncol 2013; 24(04):1087-1093. Doi: 10.1093/annonc/mds587 